\title{
Apatinib for molecular targeted therapy in tumor
}

This article was published in the following Dove Press journal:

Drug Design, Development and Therapy

13 November 2015

Number of times this article has been viewed

\section{Haijun Zhang}

Department of Oncology, Zhongda Hospital, Medical School, Southeast University, Nanjing, People's Republic of China
Correspondence: Haijun Zhang Department of Oncology, Zhongda Hospital, Medical School, Southeast University, 87 Dingjiaqiao, Nanjing 210009, People's Republic of China Tel +86 2583275418

$\mathrm{Fax}+862583275418$

Email zhanghaijunseu@I63.com
Abstract: As tumor angiogenesis is one of the hallmarks of cancer, the inhibition of vascular endothelial growth factor signaling has become an attractive anticancer approach. Apatinib, a small-molecule inhibitor of vascular endothelial growth factor receptor-2, has demonstrated encouraging anticancer activity across a broad range of malignancies, including gastric cancer, non-small-cell lung cancer, breast cancer, and hepatocellular carcinoma. In this up-to-date review, focus is not only on the structure, mechanisms, and pharmacokinetics of apatinib, but also on summarizing clinical trials and making recommendations of apatinib for patients with advanced solid tumors.

Keywords: apatinib, angiogenesis, vascular endothelial growth factor receptor-2, molecular targeted therapy, tumor

\section{Introduction}

Angiogenesis is an essential step in tumor growth and metastasis. ${ }^{1-5}$ Vascular endothelial growth factor (VEGF) signaling plays an important role in angiogenesis, and vascular endothelial growth factor receptors (VEGFRs) are tyrosine kinases functioning as key regulators of this process. Therefore, they may represent potential targets for anticancer therapy. The VEGFR family proteins consist of VEGFR-1 (FMS-like tyrosine kinase [FLT]-1), VEGFR-2 (KDR/Flk-1), and VEGFR-3 (FLT-4). ${ }^{6}$ Among them, VEGFR-2 is the principal mediator of the VEGF-induced angiogenic signaling. Thus, as a novel target, the blockage of VEGFR-2 could be a promising strategy to inhibit tumor-induced angiogenesis. ${ }^{7}$ Various VEGFR-2 inhibitors, including receptor-specific antibodies and low molecular weight chemicals such as sorafenib, vandetanib, cediranib, and sunitinib have recently been developed..$^{8-10}$ Moreover, apatinib is a small molecule that may specifically bind VEGFR-2, which could inhibit VEGF-stimulated endothelial cell migration and proliferation and decrease tumor microvascular density. ${ }^{11,12}$

Apatinib, also known as YN968D1, is one of the latest orally antiangiogenic agents with encouraging preclinical and clinical data in the treatment of a variety of solid tumors. It was approved and launched in People's Republic of China in 2014 as a subsequent-line treatment for patients with advanced gastric cancer (AGC). In addition, it is also currently undergoing Phase II/III clinical trials in People's Republic of China for the treatment of many cancer types, such as non-small-cell lung cancer (NSCLC), breast cancer, and hepatocellular carcinoma. These clinical trials demonstrate that apatinib has potential antitumor activity across a broad range of advanced solid tumors.

In this review, the structure, mechanisms, and pharmacokinetics of apatinib are addressed. Clinical trials are also summarized and recommendations are made of apatinib for patients with AGC and other cancer types. 


\section{Structure and mechanism}

Apatinib is a small-molecule antiangiogenic agent (molecular weight $493.58 \mathrm{Da}$ ) that selectively inhibits VEGFR-2 and also mildly inhibits c-Kit and c-Src tyrosine kinases. ${ }^{11}$ The molecular formula of apatinib is C25H27N5O4S, and chemical formula is $N$-[4-(1-cyano-cyclopentyl) phenyl]-2-(4-pyridylmethyl) amino-3-pyridine carboxamide, which is shown in Figure 1. As is well known, VEGF signaling plays an important role in the angiogenic process of solid tumors. When stimulated by VEGF, VEGFR-2 is auto-phosphorylated at the carboxy terminal tail and kinaseinsert region, which is the most pro-angiogenic effect. ${ }^{11,13}$ The phosphorylation of specific sites creates binding sites for the $\mathrm{SH}_{2}$ domains of various signaling molecules and has subsequent effects of cell proliferation, migration, permeability, and survival on the vascular endothelium..$^{711}$ Different tyrosine kinase inhibitors of VEGFRs do not have the same spectrum of molecular targets: apatinib with VEGFR-2, c-Kit, Ret, and c-Src; sorafenib with VEGFR$1 / 2 / 3$, platelet-derived growth factor receptor (PDGFR)- $\beta$, c-Kit, FLT-3, and Ret; sunitinib with VEGFR-1/2/3, PDGFR- $\alpha / \beta$, c-Kit, FLT-3, and Ret; pazopanib with VEGFR-1/2/3, PDGFR- $\alpha / \beta$, fibroblast growth factor receptor-1/2, and c-Kit; axitinib with VEGFR-1/2/3; tivozanib with VEGFR-1/2/3; and vandetanib with VEGFR-2, epidermal growth factor receptor (EGFR)-2, and Ret. ${ }^{14} \mathrm{By}$ binding to VEGFR-2, apatinib inhibits the effects of VEGF binding and subsequent VEGFR-2 autophosphorylation. ${ }^{14}$ In addition, apatinib-mediated VEGFR-2 inhibition also appears to inhibit downstream phosphorylated extracellular signal-regulated kinase. Through this inhibition, apatinib plays antiangiogenic and antitumor roles. ${ }^{14}$ Figure 2 schematically illustrates the possible mechanism of apatinib as the inhibitor of VEGFR-2.

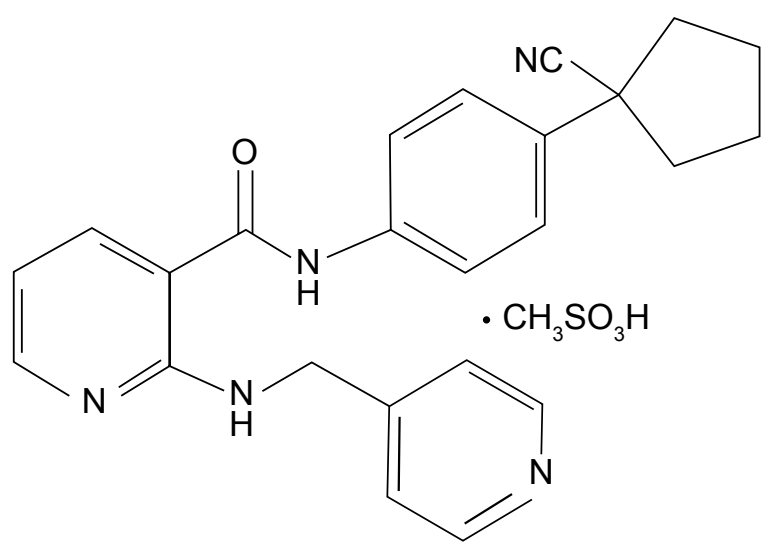

Figure I Chemical structure of apatinib.

\section{Pharmacokinetics}

Pharmacokinetic analysis by $\mathrm{Li}$ et al showed that the time to maximum plasma concentration level was $\sim 4$ hours after dose and the mean half-life was 9 hours. ${ }^{12}$ In three separate dosing groups, $500 \mathrm{mg}(\mathrm{n}=8), 750 \mathrm{mg}(\mathrm{n}=12)$, and $800 \mathrm{mg}$ $(n=8)$, of 28 patients enrolled into a single dose arm, the $\mathrm{C}_{\max }$ and $\mathrm{AUC}_{24}$ values were dose-dependent and demonstrated variability among patients with $\mathrm{C}_{\max }$ of 1,521, 2,379, and 2,833 ng/mL and $\mathrm{AUC}_{24}$ of 11,295, 18,172, and $21,975 \mathrm{ng} \cdot \mathrm{h} / \mathrm{mL}$, respectively. ${ }^{8}$ Steady-state conditions analysis of eleven patients enrolled in the multidose cohort suggested no accumulation during 56 days once a day administration of $750 \mathrm{mg}$ apatinib. ${ }^{12}$ The primary routes of apatinib biotransformation include $E$ - and $Z$-cyclopentyl3-hydroxylation, $N$-dealkylation, pyridyl-25- $N$-oxidation, 16-hydroxylation, dioxygenation, and $O$-glucuronidation after 3-hydroxylation. ${ }^{15}$ Nine major metabolites were confirmed, of which $E$-3-hydroxy-apatinib-O-glucuronide (M9-2) was the major circulating metabolite. ${ }^{11}$ The total recovery of the administered dose was $76.8 \%$ within 96 hours postdose, with $69.8 \%$ and $7.02 \%$ of the administered dose excreted in feces and urine, respectively. ${ }^{15}$

\section{Preclinical research}

Several basic studies have been dedicated to the antitumor activity of apatinib in vitro and in vivo. In vitro, apatinib potently suppressed the kinase activities of VEGFR-2, c-Kit, and c-Src, and inhibited cellular phosphorylation of VEGFR-2, c-Kit, and PDGFRb. ${ }^{11}$ Apatinib could also effectively inhibit proliferation, migration, and tube formation of human umbilical vein endothelial cells induced by fetal bovine serum, and block the budding of rat aortic ring. ${ }^{11}$ Encouraged by the remarkable inhibitory activity against VEGFR-2 tyrosine kinase in vitro, Tian et al further performed the investigation of the potential antitumor effect of apatinib in vivo. ${ }^{11}$ The results demonstrated that apatinib showed antitumor efficacy in vivo when administrated alone or in combination with chemotherapy against a variety of established tumor xenografts with good tolerance. ${ }^{11}$ To date, the outcome of cancer chemotherapy still encounters two major challenges: nonspecific targets and multidrug resistance (MDR). In fact, more than $90 \%$ of patients with malignant tumors die of MDR. ${ }^{16,17}$ Apatinib could also reverse cancer MDR mediated by MDR protein 1 (ABCB1), MDRassociated protein 1 (MARP1), and breast cancer resistant protein (BCRP) through inhibiting their transport function as well. ${ }^{18,19}$ Thus, apatinib may be useful in overcoming MDR to other conventional antineoplastic drugs. 


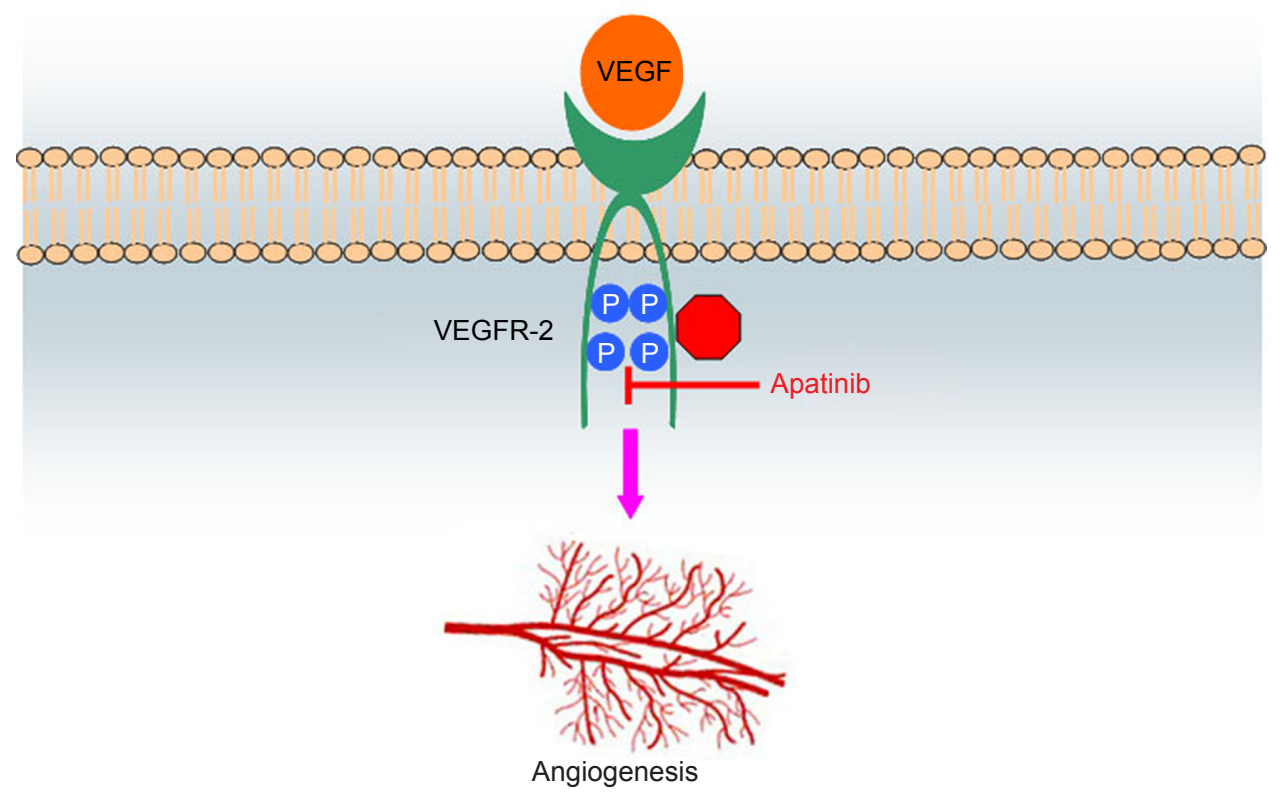

Figure 2 Schematic illustration of the possible mechanism of apatinib as the inhibitor of VEGFR-2.

Notes: By specifically binding to the phosphorylation sites of VEGFR-2, apatinib inhibits the subsequent effects on the vascular endothelium, including cell proliferation, migration, permeability, and survival. Through this inhibition, apatinib plays an antiangiogenic role.

Abbreviations: VEGFR-2, vascular endothelial growth factor receptor-2; VEGF, vascular endothelial growth factor.

\section{Clinical trials of apatinib for molecular targeted therapy in tumor}

The efficacy of apatinib for the treatment of a variety of solid tumors has been investigated in several clinical trials, including various Phase I, II, and III studies on registry, which is summarized in Table 1.

\section{Phase I}

Phase I study of apatinib as an inhibitor of angiogenesis by $\mathrm{Jin} \mathrm{Li}$ as principal investigator was conducted to determine the maximum tolerated dose, safety profile, pharmacokinetic variables, and antitumor activity in advanced solid malignancies (NCT00633490). ${ }^{12}$ In this study, 46 patients with advanced cancer were enrolled, including 34 cases of gastrointestinal cancer, three cases of lung cancer, three cases of breast cancer, and six cases of other tumor types. The maximum tolerated dose was determined to be $850 \mathrm{mg}$ qd. The recommended dose was $750 \mathrm{mg}$ qd, which could be well tolerated. Besides, among 37 evaluable patients, partial response was noted in seven patients (18.9\%) and stable disease in 24 (64.9\%), with a disease control rate (DCR) of $83.8 \%$ at 8 weeks. The safety profile was acceptable and the regimen was also

Table I Characteristics of the clinical trials of apatinib for molecular targeted therapy in tumor

\begin{tabular}{|c|c|c|c|c|}
\hline Study & Trial & Design & Tumor type & Outcomes (apatinib vs placebo) \\
\hline Li et al ${ }^{12}$ & Phase I & Dose escalation & $\begin{array}{l}\text { Advanced solid } \\
\text { tumors }\end{array}$ & $\begin{array}{l}\text { MTD: } 850 \text { mg qd } \\
\text { Recommended dose: } 750 \text { mg qd }\end{array}$ \\
\hline Li et $\mathrm{al}^{22}$ & Phase II & $\begin{array}{l}\text { Randomized, placebo-controlled, } \\
\text { parallel-arm }\end{array}$ & Gastric cancer & $\begin{array}{l}\text { mPFS: } 3.67^{\mathrm{a}} \text { vs } 1.40 \text { months } \\
\text { mPFS: } 3.20^{\mathrm{b}} \text { vs } 1.40 \text { months } \\
\text { mOS: } 4.83^{\mathrm{a}} \text { vs } 2.50 \text { months } \\
\text { mOS: } 4.27^{\mathrm{b}} \text { vs } 2.50 \text { months }\end{array}$ \\
\hline Hu et $\mathrm{al}^{25}$ & Phase II & $\begin{array}{l}\text { Prospective, open-label, } \\
\text { single arm }\end{array}$ & $\begin{array}{l}\text { Triple-negative } \\
\text { breast cancer }\end{array}$ & $\begin{array}{l}\text { mPFS: } 3.3 \text { months } \\
\text { mOS: } 10.6 \text { months }\end{array}$ \\
\hline Hu et $\mathrm{al}^{24}$ & Phase II & $\begin{array}{l}\text { Multicenter, open-label, } \\
\text { single arm }\end{array}$ & $\begin{array}{l}\text { Non-triple-negative } \\
\text { breast cancer }\end{array}$ & $\begin{array}{l}\text { mPFS: } 4.0 \text { months } \\
\text { mOS: } 10.3 \text { months }\end{array}$ \\
\hline Zhang et $\mathrm{al}^{33}$ & Phase II & $\begin{array}{l}\text { Multicenter, randomized, } \\
\text { placebo-controlled }\end{array}$ & $\begin{array}{l}\text { Non-small-cell lung } \\
\text { cancer }\end{array}$ & mPFS: 4.7 vs 1.9 months \\
\hline Qin $^{34}$ & Phase III & $\begin{array}{l}\text { Randomized, multicenter, } \\
\text { double-blind, placebo-controlled }\end{array}$ & Gastric cancer & $\begin{array}{l}\text { mOS: } 195 \text { vs } 140 \text { days } \\
\text { mPFS: } 78 \text { vs } 53 \text { days }\end{array}$ \\
\hline
\end{tabular}

Notes: ${ }^{\text {a }}, 850 \mathrm{mg}$ of apatinib qd; ' , apatinib $425 \mathrm{mg}$ bid.

Abbreviations: MTD, maximum tolerated dose; mPFS, median progression-free survival; mOS, median overall survival; bid, twice a day; qd, once a day. 
well tolerated. The most frequently observed drug-related adverse events were hypertension $(69.5 \%, 29$ grade $1-2$ and three grade $3-4)$, proteinuria $(47.8 \%, 16$ grade $1-2$ and six grade $3-4)$, and hand-foot syndrome $(45.6 \%, 15$ grade $1-2$ and six grade 3-4).

\section{Phase II}

\section{Apatinib for AGC/mGC}

Gastric cancer is the third most common cause of cancerrelated death in the world. ${ }^{20,21}$ Although there is a better chance of recovery in early gastric cancer, the prognosis of patients with metastatic or AGC remains poor, with a median overall survival (mOS) of 1 year. ${ }^{20,21}$ There may be clinical remission or disease stabilization in AGC patients who receive first-line chemotherapy; however, most will ultimately experience disease progression, and thus effective second-line chemotherapy is essential. However, after failure of second-line chemotherapy, the outcomes of further treatment are poor, yielding response rates of $0 \%-5 \%$ with no evidence of prolonged survival. ${ }^{20}$ Therefore, novel and more effective treatment options are urgently needed to provide survival benefit for patients with AGC or metastatic gastric cancer (mGC). Fortunately, apatinib is a new therapy option providing hope for patients with AGC or $\mathrm{mGC}$ who have previously failed second-line chemotherapy. ${ }^{11}$ In a randomized, placebo-controlled, parallel-arm Phase II trial (NCT00970138), Li et al evaluated apatinib as a subsequentline therapy for patients with histologically confirmed AGC or $\mathrm{mGC}^{22}$ In the trial, 144 enrolled patients were divided into three groups: placebo (group A), $850 \mathrm{mg}$ of apatinib qd (group B), and apatinib $425 \mathrm{mg}$ bid (group C). Progressionfree survival (PFS) was the primary end point. Secondary end points included DCR, objective response rate, overall survival, and quality of life. The outcomes demonstrated that the median progression-free survival (mPFS) for groups A, $\mathrm{B}$, and $\mathrm{C}$ was 1.40 months (95\% confidence interval [CI]: 1.20-1.83 months), 3.67 months (95\% CI: 2.17-6.80 months), and 3.20 months (95\% CI: 2.37-4.53 months), respectively. mOS for groups A, B, and C was 2.50 months $(95 \% \mathrm{CI}$ : 1.87-3.70 months), 4.83 months (95\% CI: 4.03-5.87 months), and 4.27 months (95\% CI: 3.83-4.77 months), respectively. There were statistically significant differences between the apatinib groups and the placebo group in PFS and overall survival $(P<0.001)$. But, there was no significant difference between apatinib $425 \mathrm{mg}$ bid vs $850 \mathrm{mg}$ qd (hazard ratio [HR]:1.22; 95\% CI: $0.68-2.20 ; P<0.551)$. These results illustrated that apatinib showed improved PFS and overall survival in heavily pretreated patients with AGC or mGC who had experienced treatment failure with two or more chemotherapy regimens.

\section{Apatinib for TNBC and non-TNBC}

Breast cancer is the most common malignancy in women worldwide. ${ }^{23}$ Although 5-year recurrence rates and 15-year mortality rates could benefit from effective chemotherapy and hormonal therapy for early breast cancer, many patients still experience tumor relapse or metastasis. ${ }^{24}$ In addition to chemotherapy, endocrine therapy or targeted human EGFR-2 therapy plays an important role of the therapy for patients with metastatic non-triple-negative breast cancer (TNBC); however, most patients will eventually develop drug resistance. ${ }^{24}$ Metastatic TNBC is also particularly challenging due to the lack of recognized therapeutic molecular biology targets, which is highly proliferative, with a high risk for recurrence, relatively poorer prognosis, and rapid disease progression. ${ }^{25}$ Novel drugs for such patients with metastatic breast cancer are therefore needed urgently. To solve it, a prospective, open-label, Phase II trial by $\mathrm{Hu}$ et al aimed to evaluate the efficacy and safety of apatinib in heavily pretreated patients with metastatic TNBC (NCT01176669). ${ }^{25}$ In the trial, after the optimum dose level of $500 \mathrm{mg} /$ day was recommended by Phase IIa, a Phase IIb study of 59 patients with metastatic TNBC was activated, with the endpoint PFS. ${ }^{25}$ The outcomes were reported that mPFS and mOS were 3.3 months (95\% CI: 1.7-5.0 months) and 10.6 months (95\% CI: 5.6-15.7 months), respectively. In the 56 evaluable patients, overall response and clinical benefit rates were $10.7 \%$ and $25.0 \%$, respectively. As for adverse events, the most common grade $3 / 4$ hematologic toxicities were thrombocytopenia (13.6\%), leukopenia $(6.8 \%)$, neutropenia $(3.4 \%)$, and anemia $(1.7 \%)$. The most frequent grade 3/4 nonhematologic toxicities were hand-foot syndrome, proteinuria, hypertension, and increased alanine aminotransferase. In the last decade, several multiple kinase inhibitors of VEGFR, such as sunitinib and sorafenib, have been developed for the antiangiogenic therapy of TNBC. There is minor difference when apatinib is compared with these multiple kinase inhibitors while sunitinib and sorafenib have demonstrated modest single-agent activity but improved outcomes combined with chemotherapy. ${ }^{26-29}$ As for the efficacy of apatinib on heavily pretreated, metastatic non-TNBC, another multicenter, open-label, single arm, Phase II trial study was performed. ${ }^{24}$ Inclusion criteria included patients who failed in the metastatic setting of at least one and at most four prior chemotherapy regimens, 
hormone receptor-positive patients who received at least one endocrine drug, and human EGFR-2-positive patients who were treated with at least one anti-human EGFR-2 drug. ${ }^{24}$ There were 38 enrolled patients who received apatinib for a median of 4 cycles (range from 0 to 10 cycles). In the study, mPFS was 4.0 months (95\% CI: 2.8-5.2 months) and mOS was 10.3 months (95\% CI: 9.1-11.6 months). In all, 36 patients were eligible for efficacy analysis, with an objective response rate of $16.7 \%(6 / 36)$ and DCR of $66.7 \%$ (24/36). Adverse events were also observed, and the most common grade 3 or 4 nonhematologic ones were hypertension (20.5\%), hand-foot syndrome (10.3\%), and proteinuria $(5.1 \%)$.

\section{Apatinib for advanced NSCLC}

Lung cancer is a leading cause of cancer-related mortality that accounts for $13 \%$ and $18 \%$ of all cancer-related cases and deaths, respectively, in the world. ${ }^{30}$ Approximately, $80 \%-85 \%$ of lung cancer cases are NSCLC. Unfortunately, $75 \%$ of NSCLC patients present with locally advanced or metastatic disease at the time of diagnosis. ${ }^{31}$ Although surgical excision and chemotherapy are attainable in some patients with advanced NSCLC, the therapeutic options for locally advanced or metastatic disease remain limited. ${ }^{32}$ In recent years, targeted drugs shift the traditional treatment mode of NSCLC, including apatinib. A multicenter, randomized, placebo-controlled, Phase II trial by Zhang et al was to determine whether apatinib could improve PFS compared with placebo in patients with advanced nonsquamous NSCLC who failed two lines of treatment (NCT01270386). ${ }^{33}$ In the study, 135 patients were randomized in a 2:1 fashion to apatinib $750 \mathrm{mg}$ qd vs placebo until disease progression or unacceptable toxicity. As expected, mPFS was 4.7 months for apatinib group vs that of 1.9 months for placebo group with an HR of 0.278 (95\% CI: $0.170-0.455)(P<0.0001)$. The response rate and DCR were also significantly better in apatinib arm (12.2\% and 68.9\%) than in placebo arm ( $0 \%$ and $24.4 \%)(P=0.0158$ and $P<0.0001)$. Although hypertension, proteinuria, and hand-foot syndrome were the most frequent adverse events, they were generally mild or moderate in severity and were manageable..$^{33}$ In summary, the Phase II trial shows that apatinib has substantial clinical activity without significant additional toxicity in patients with advanced NSCLC.

\section{Phase III}

As illustrated above, apatinib provides a new treatment option and leads a new hope for patients with advanced or
mGC. Further study was carried out based on Phases I and II. A randomized, multicenter, double-blind, placebo-controlled Phase III trial by Qin investigated single-agent apatinib in patients with AGC who previously failed to second-line chemotherapy (NCT01512745). ${ }^{34}$ Patients were randomized in a 2:1 fashion to apatinib $850 \mathrm{mg}$ qd or placebo with a planned total enrollment of 270 patients. As for the efficacy, patients treated with apatinib at the dose of $850 \mathrm{mg}$ qd had significantly prolonged $\operatorname{mOS}$ (195 vs 140 days; $\mathrm{HR}=0.71$, 95\% CI: $0.54-0.94 ; P<0.016)$ and mPFS (78 vs 53 days; HR $=0.44$, 95\% CI: 0.33-0.61; $P<0.0001)$ compared with those treated with placebo. The objective response rate of apatinib group and placebo group were $2.84 \%$ and $0.00 \%$, respectively. Hypertension, hand-foot syndrome, proteinuria, fatigue, anorexia, and elevated aminotransferase were grade $3 / 4$ adverse reactions observed in more than $2 \%$ of patients, which could be managed by dose interruptions or reductions. Given the excellent results obtained in the Phase III trial, apatinib offers a new treatment option for patients with advanced $\mathrm{mGC} .^{21,34}$

\section{Ongoing trials}

Currently, there are also multiple ongoing clinical trials investigating apatinib for molecular targeted therapy in tumor. For example, a double-blind, placebo-controlled, Phase III study explores apatinib for patients with EGFR wild-type nonsquamous NSCLC (NCT 02332512). Phase II and Phase III trials investigating apatinib in subsequentline treatment for hepatocellular carcinoma are also actively recruiting (NCT02329860).

\section{Side effects of apatinib treatment}

Apatinib is generally well tolerated by patients. However, adverse effects also occurred, and these are considered manageable. The most frequently observed drug-related adverse events were hypertension, proteinuria, and hand-foot syndrome. Management of these toxicities could include dose reduction, interruption, or termination.

\section{Conclusion and future directions for drug development}

Apatinib is a novel, orally bioavailable small-molecule tyrosine kinase inhibitor of VEGFR-2 that appears to be a promising agent in the treatment of a variety of tumor types, and has demonstrated improved outcomes and a tolerable safety profile in subsequent-line therapy. Since single modality has not always been sufficiently effective, combination therapies are the solution to improve 
the outcome of treatment. However, Lin et al reported that apatinib combined with elemene injection (a Chinese medicine) for the treatment of $\mathrm{H} 22$ solid tumor in mice resulted in worse effectiveness than apatinib alone. ${ }^{35}$ Thus, the multiple therapeutics strategies of apatinib combined with other therapies, such as chemotherapy and radiotherapy, should be implemented and developed in future clinical trials to address these issues. Besides, to find biomarkers to predict drug efficacy is also one of the challenges with antiangiogenic therapies using apatinib. Predictive biomarkers could discriminate patients who are most likely to be sensitive to apatinib and avoid exposure to useless toxic medicine. ${ }^{36}$ Thus, additional studies into biomarkers may be useful in predicting personalized therapeutic response.

\section{Acknowledgments}

This work was supported by the National Natural Science Foundation of China $(31200750,81371678)$ and the Natural Science Foundation of Jiangsu Province (BK2012332).

\section{Disclosure}

The author reports no conflicts of interest in this work.

\section{References}

1. Cowey CL. Profile of tivozanib and its potential for the treatment of advanced renal cell carcinoma. Drug Des Devel Ther. 2013; 7:519-527.

2. Chimote G, Sreenivasan J, Pawar N, Subramanian J, Sivaramakrishnan H, Sharma S. Comparison of effects of anti-angiogenic agents in the zebrafish efficacy-toxicity model for translational anti-angiogenic drug discovery. Drug Des Devel Ther. 2014;8:1107-1123.

3. Yoshida H, Yabuno A, Fujiwara K. Critical appraisal of bevacizumab in the treatment of ovarian cancer. Drug Des Devel Ther. 2015;9: 2351-2358.

4. Iman $\mathrm{V}$, Karimian $\mathrm{H}$, Mohan $\mathrm{S}$, et al. In vitro and in vivo anti-angiogenic activity of girinimbine isolated from Murraya koenigii. Drug Des Devel Ther. 2015;9:1281-1292.

5. Fontanella C, Ongaro E, Bolzonello S, Guardascione M, Fasola G, Aprile G. Clinical advances in the development of novel VEGFR2 inhibitors. Ann Transl Med. 2014;2(12):123.

6. Ding J, Chen X, Dai X, Zhong D. Simultaneous determination of apatinib and its four major metabolites in human plasma using liquid chromatography-tandem mass spectrometry and its application to a pharmacokinetic study. J Chromatogr B Analyt Technol Biomed Life Sci. 2012;895-896:108-115.

7. Hamerlik P, Lathia JD, Rasmussen R, et al. Autocrine VEGF-VEGFR2Neuropilin-1 signaling promotes glioma stem-like cell viability and tumor growth. J Exp Med. 2012;209(3):507-520.

8. Wedge SR, Ogilvie DJ, Dukes M, et al. ZD4190: an orally active inhibitor of vascular endothelial growth factor signaling with broad-spectrum antitumor efficacy. Cancer Res. 2000;60(4):970-975.

9. Wood JM, Bold G, Buchdunger E, et al. PTK787/ZK 222584, a novel and potent inhibitor of vascular endothelial growth factor receptor tyrosine kinases, impairs vascular endothelial growth factor-induced responses and tumor growth after oral administration. Cancer Res. 2000;60(8):2178-2189.
10. Clark JW, Eder JP, Ryan D, Lathia C, Lenz HJ. Safety and pharmacokinetics of the dual action Raf kinase and vascular endothelial growth factor receptor inhibitor, BAY 43-9006, in patients with advanced, refractory solid tumors. Clin Cancer Res. 2005;11(15):5472-5480.

11. Tian S, Quan H, Xie C, et al. YN968D1 is a novel and selective inhibitor of vascular endothelial growth factor receptor-2 tyrosine kinase with potent activity in vitro and in vivo. Cancer Sci. 2011; 102(7):1374-1380.

12. Li J, Zhao X, Chen L, et al. Safety and pharmacokinetics of novel selective vascular endothelial growth factor receptor-2 inhibitor YN968D1 in patients with advanced malignancies. BMC Cancer. 2010;10:529.

13. Holmes K, Roberts OL, Thomas AM, Cross MJ. Vascular endothelial growth factor receptor-2: structure, function, intracellular signalling and therapeutic inhibition. Cell Signal. 2007;19(10):2003-2012.

14. Scott AJ, Messersmith WA, Jimeno A. Apatinib: a promising oral antiangiogenic agent in the treatment of multiple solid tumors. Drugs Today (Barc). 2015;51(4):223-239.

15. Ding J, Chen X, Gao Z, et al. Metabolism and pharmacokinetics of novel selective vascular endothelial growth factor receptor-2 inhibitor apatinib in humans. Drug Metab Dispos. 2013;41(6):1195-1210.

16. Zhang H, Xiong J, Guo L, Patela N, Guang X. Integrated traditional Chinese and western medicine modulator for overcoming the multidrug resistance with carbon nanotubes. RSC Adv. 2015;5(87): 71287-71296.

17. Wu P, Li S, Zhang H. Design real-time reversal of tumor multidrug resistance cleverly with shortened carbon nanotubes. Drug Des Devel Ther. 2014;8:2431-2438.

18. Tong XZ, Wang F, Liang S, et al. Apatinib (YN968D1) enhances the efficacy of conventional chemotherapeutical drugs in side population cells and ABCB1-overexpressing leukemia cells. Biochem Pharmacol. 2012;83(5):586-597.

19. Mi YJ, Liang YJ, Huang HB, et al. Apatinib (YN968D1) reverses multidrug resistance by inhibiting the efflux function of multiple ATPbinding cassette transporters. Cancer Res. 2010;70(20):7981-7991.

20. Liu L, Yu H, Huang L, et al. Progression-free survival as a surrogate endpoint for overall survival in patients with third-line or later-line chemotherapy for advanced gastric cancer. Onco Targets Ther. 2015;8: 921-928.

21. Geng R, Li J. Apatinib for the treatment of gastric cancer. Expert Opin Pharmacother. 2015;16(1):117-122.

22. Li J, Qin S, Xu J, et al. Apatinib for chemotherapy-refractory advanced metastatic gastric cancer: results from a randomized, placebo-controlled, parallel-arm, phase II trial. J Clin Oncol. 2013;31(26):3219-3225.

23. Althuis MD, Dozier JM, Anderson WF, Devesa SS, Brinton LA. Global trends in breast cancer incidence and mortality 1973-1997. Int J Epidemiol. 2005;34(2):405-412.

24. Hu X, Cao J, Hu W, et al. Multicenter phase II study of apatinib in non-triple-negative metastatic breast cancer. BMC Cancer. 2014; $14: 820$.

25. Hu X, Zhang J, Xu B, et al. Multicenter phase II study of apatinib, a novel VEGFR inhibitor in heavily pretreated patients with metastatic triple-negative breast cancer. Int J Cancer. 2014;135(8):1961-1969.

26. Clark O, Botrel TE, Paladini L, Ferreira MB. Targeted therapy in triple-negative metastatic breast cancer: a systematic review and metaanalysis. Core Evid. 2014;9:1-11.

27. Bramati A, Girelli S, Torri V, et al. Efficacy of biological agents in metastatic triple-negative breast cancer. Cancer Treat Rev. 2014; 40(5):605-613.

28. Vasudev NS, Reynolds AR. Anti-angiogenic therapy for cancer: current progress, unresolved questions and future directions. Angiogenesis. 2014;17(3):471-494.

29. Santoni M, Conti A, Massari F, et al. Treatment-related fatigue with sorafenib, sunitinib and pazopanib in patients with advanced solid tumors: an up-to-date review and meta-analysis of clinical trials. Int $J$ Cancer. 2015;136(1):1-10.

30. Jemal A, Bray F, Center MM, Ferlay J, Ward E, Forman D. Global cancer statistics. CA Cancer J Clin. 2011;61:69-90. 
31. Siegel R, Ma J, Zou Z, Jemal A. Cancer statistics. CA Cancer J Clin. 2014;64:9-29.

32. Zhong A, Xiong X, Shi M, Xu H. The efficacy and safety of pemetrexed-based doublet therapy compared to pemetrexed alone for the second-line treatment of advanced non-small-cell lung cancer: an updated meta-analysis. Drug Des Devel Ther. 2015;9:3685-3693.

33. Zhang L, Shi M, Huang C, et al. A phase II, multicenter, placebocontrolled trial of apatinib in patients with advanced nonsquamous non-small cell lung cancer (NSCLC) after two previous treatment regimens. J Clin Oncol. 2012;30(Suppl):Abst 7548.

34. Qin S. Phase III study of apatinib in advanced gastric cancer: a randomized, double-blind, placebo-controlled trial. J Clin Oncol. 2014; 32(Suppl):Abst 4003.
35. Lin Y, Wang K, Hu C, Lin L, Qin S, Cai X. Elemene injection induced autophagy protects human hepatoma cancer cells from starvation and undergoing apoptosis. Evid Based Complement Alternat Med. 2014; 2014:637528.

36. Fan M, Zhang J, Wang Z, et al. Phosphorylated VEGFR2 and hypertension: potential biomarkers to indicate VEGF-dependency of advanced breast cancer in anti-angiogenic therapy. Breast Cancer Res Treat. 2014; 143(1):141-151.

\section{Publish your work in this journal}

Drug Design, Development and Therapy is an international, peerreviewed open-access journal that spans the spectrum of drug design and development through to clinical applications. Clinical outcomes, patient safety, and programs for the development and effective, safe, and sustained use of medicines are a feature of the journal, which has also been accepted for indexing on PubMed Central. The manuscript management system is completely online and includes a very quick and fair peer-review system, which is all easy to use. Visit http://www.dovepress.com/testimonials.php to read real quotes from published authors.

\footnotetext{
Submit your manuscript here: http://www.dovepress.com/drug-design-development-and-therapy-journal
} 